\title{
A PARTITION RESULT FOR ALGEBRAIC VARIETIES
}

\author{
ANER SHALEV \\ (Communicated by William Adams)
}

\begin{abstract}
Let $K$ be a finite field. It is shown that, given positive integers $d$ and $r$, there exists $M=M(d, r)$, such that any variety $V=V(f) \subseteq K^{n}$, defined by a polynomial $f$ of degree $d$ in $n \geq M$ variables over $K$, can be partitioned into affine subspaces, each of dimension $r$. This result, relying on a theorem of R. Brauer, holds in fact for many other fields, including algebraically closed fields. It may provide a partial structural explanation to a divisibility phenomenon discovered by J. Ax.
\end{abstract}

\section{MAin Result}

Let $K$ be a field with $q=p^{k}$ elements. The classical Chevalley-Warning theorem, proved in 1936, states that if $f=f(\bar{x})$ is polynomial in $n$ variables over $K$ of degree $d<n$, then $N(f)$-the number of zeros of $f$ in $K^{n}$-is divisible by $p([4,9]$; see also $[5,7])$.

This result was remarkably extended by J. Ax in 1964 [2].

Theorem (Ax). Suppose $f \in K\left[x_{1}, \ldots, x_{n}\right], \operatorname{deg}(f)=d$. Let $r$ be a positive integer such that $n>d \cdot r$. Then $q^{r}$ divides $N(f)$.

This theorem is the best possible, in the sense that the condition on $n$ cannot be weakened. Ax also formulated a divisibility result for a system of polynomial equations, which was later sharpened by N. M. Katz [6]. Recently, D. Wan [8] gave a more elementary proof of Katz's theorem, avoiding the use of the $p$-adic theory of zeta functions. See also [1] for more refined divisibility properties, relying on the "geometry" of the monomials appearing in $f$ and not only on the parameters $n$ and $d$.

The theorem of $\mathrm{Ax}$ shows that the number of (rational) points in varieties defined by a polynomial of fixed degree in a sufficiently large number of variables, is divisible by arbitrarily large $q$ th-powers, where $q$ is the order of the ground field.

It is natural to ask whether there exists any "hidden structure" behind this phenomenon. The most satisfactory structural explanation to a divisibility re-

\footnotetext{
Received by the editors September 25, 1989 and, in revised form, February 20, 1990.

1980 Mathematics Subject Classification (1985 Revision). Primary 11G25, 12E12; Secondary $14 \mathrm{~A} 10$. 
sult is the existence of a certain partition. Obviously, an affine space (i.e., a coset of a linear space) of dimension $r$ over $K$ consists of $q^{r}$ elements. Therefore, in our case one may wonder whether, for given $d$ and $r$, any variety $V(f)$, defined by a polynomial $f$ of degree $d$ in a sufficiently large number of variables, may be partitioned into $r$-dimensional affine spaces.

The purpose of this paper is to prove this hypothesis, not only for finite fields but for a large family of fields, including algebraically closed ones. However, the bounds required for divisibility are usually lower than those insuring the existence of a suitable partition.

One step in this direction was made by R. Brauer in 1945. In order to formulate this result, consider the following field-theoretical property, where $\omega$ denotes the natural numbers:

There exists a function $\phi: \omega \rightarrow \omega$ such that, if $d \in \omega$ and $n \geq \phi(d)$, then any polynomial of the form

$$
a_{1} x_{1}^{d}+a_{2} x_{2}^{d}+\cdots+a_{n} x_{n}^{d}=0
$$

over $K$ has a nontrivial zero in $K^{n}$.

It is clear that algebraically closed fields, finite fields, and in fact any $C_{i}$ field (e.g., $p$-adic fields) satisfy $(*)$.

In [3] Brauer considered a system of homogeneous equations over a field satisfying $(*)$. He proved that if the number of variables $n$ is larger than a certain function of the degrees of the equations and a given parameter $r$, then the variety defined by the equations contains an $r$-dimensional linear space. Brauer's theorem is easily modified to the nonhomogeneous case, provided that the equations have no constant terms. Indeed, one should replace every equation of degree $d$ with its homogeneous constituents of degrees $1,2, \ldots, d$ respectively. Dealing, for simplicity, with one equation, one obtains the following theorem from [3].

Theorem. Let $K$ be a field satisfying $(*)$. Then, for every $d$ and $r$, there exists $N=N(d, r)$, such that if $f$ is any polynomial of degree $d$ in $n \geq N$ variables over $K$ with $f(\overline{0})=0$, then the variety $V(f) \subseteq K^{n}$ contains an $r$-dimensional linear space.

Remark. If $f(\bar{a})=0$ for some $\bar{a} \in K^{n}$ (not necessarily $\overline{0}$ ), then, via an affine change of variables, it follows that $V(f)$ contains an $r$-dimensional affine space $U$ such that $\bar{a} \in U$. Hence Brauer's result shows that $V(f)$ may actually be covered by $r$-dimensional affine spaces, but these need not be pairwise disjoint.

We can now state and prove our main result.

Theorem 1. Let $K$ be a field satisfying (*). Then, for every $d$ and $r$ there exists $M=M(d, r)$, such that if $f$ is any polynomial of degree $d$ in $n \geq M$ variables over $K$, then the variety $V(f) \subseteq K^{n}$ can be partitioned into r-dimensional affine spaces. 
Proof. Let $N$ be the function appearing in Brauer's theorem. Define $M(d, r)$ inductively by

$$
M(1, r)=r+1 ; \quad M(d, r)=N(d, M(d-1, r)) \quad(d \geq 2) .
$$

Let us prove, by induction on $d$, that, for all $r, M(d, r)$ is as required.

The case $d=1$ is clear (since $V(f)$ itself is an affine space of dimension $r)$. Now, let $f$ be a polynomial of degree $d \geq 2$ in $n \geq M(d, r)$ variables. If $V(f)=\varnothing$ we are done (there is nothing to partition), so let us assume $V(f) \neq \varnothing$. Put $s=M(d-1, r)$. By Brauer's theorem, $V(f)$ contains an affine space $U$ of dimension $s$. After an affine change of variables we may assume that $U$ is given by the $n-s$ linear constraints $x_{s+1}=0$, $x_{s+2}=0, \ldots, x_{n}=0$. This means that the function $K^{s} \rightarrow K$ defined by the polynomial $f_{0}=f\left(x_{1}, \ldots, x_{s}, 0, \ldots, 0\right)$ is identically zero. Therefore $f_{0}$ is the zero polynomial (if $K$ is finite, we will have to assume here, as we may, that $f$ is reduced). We conclude that every monomial which appears in $f$ involves some of the vanishing variables $x_{s+1}, \ldots, x_{n}$. Hence, any substitution $x_{s+1} \leftarrow a_{s+1}, \ldots, x_{n} \leftarrow a_{n}$ (where $a_{i} \in K$ ) will reduce the degree of $f$.

Now, given $\bar{a}=\left(a_{s+1}, \ldots, a_{n}\right) \in K^{n-s}$, consider the polynomial $f_{\bar{a}}=$ $f\left(x_{1}, \ldots, x_{s}, a_{s+1}, \ldots, a_{n}\right)$. Its degree is $\leq d-1$, and its number of variables is $s=M(d-1, r)$. By induction hypothesis, $V\left(f_{\bar{a}}\right) \subseteq K^{s}$ is partitioned into affine spaces, each of dimension $r$. Letting $\bar{a} \in K^{n-s}$ take all its possible values, we get the required partition of the original variety $V(f)$.

Remark. A similar result holds for algebraic varieties defined by more than one polynomial: if the number of variables is greater than a certain function of the degrees of the defining polynomials and a given parameter $r$, then the variety obtained can be partitioned into $r$-dimensional affine spaces. The proof is essentially the same.

\section{NUMERICAL ESTIMATES}

In this section we consider the problem of the evaluation of the functions $N_{K}(d, r)$ and $M_{K}(d, r)$ associated with a finite field $K$, defined by

$N_{K}(d, r)=\min \left\{n:\right.$ if $f \in K\left[x_{1}, \ldots, x_{n}\right], \operatorname{deg}(f) \leq d$, and $V(f) \neq \varnothing$, then $V(f)$ contains an $r$-dimensional affine space $\}$.

$$
M_{K}(d, r)=\min \left\{n: \text { if } f \in K\left[x_{1}, \ldots, x_{n}\right] \text { and } \operatorname{deg}(f) \leq d \text {, then } V(f)\right. \text { may }
$$
be partitioned into $r$-dimensional affine spaces $\}$.

Although we shall not get much information on the behaviour of $M_{K}(d, r)$ (except for a certain astronomical upper bound), our estimates for $N_{K}(d, r)$ are more satisfactory; they show that, for a finite field $K$ and fixed $d<|K|$, $N(d, r)=O\left(r^{d-1}\right)$. Similar estimations may be carried out for algebraically closed fields as well. 
It should be noted that Brauer's proof does not include any specific evaluation of the function $N(d, r)$; however, in our estimation we apply some of his ideas. We also use Warning's second theorem [9], showing that if $|K|=q$, then any system of equations in $n$ variables over $K$, whose sum of degrees is $d$, has at least $q^{n-d}$ solutions, provided it has a solution.

Theorem 2. Suppose $d<|K|<\infty$. Then for all $r$,

$$
\frac{1}{d}\left(\begin{array}{c}
r+d \\
d-1
\end{array}\right)+r-1<N_{K}(d, r) \leq\left(\begin{array}{c}
r+d \\
d-1
\end{array}\right)+r .
$$

Consequently, fixing $d$, we obtain $N_{K}(d, r)=O\left(r^{d-1}\right)$.

Proof. We first prove the upper bound.

Let $f$ be a polynomial of degree $\leq d$ in $n$ variables, and suppose $n \geq$ $\left(\begin{array}{c}r+d \\ d-1\end{array}\right)+r$. We assume, by induction, that $V(f)$ contains an affine space $\overline{U^{\prime}}$ of dimension $r-1$, and we would like to extend it to an $r$-dimensional affine space $U$, lying in $V(f)$. We may assume that $\overline{0} \in U^{\prime}$ (so that $U^{\prime}$ is a linear space).

Let $\bar{u}_{1}, \ldots, \bar{u}_{r-1}$ be a basis for $U^{\prime}$. We introduce $r$ new variables $y_{1}, \ldots$, $y_{r}$ and form the expression

$$
f\left(y_{1} \bar{u}_{1}+\cdots+y_{r-1} \bar{u}_{r-1}+y_{r} \bar{x}\right)=\sum_{\bar{m}} f_{\bar{m}}(\bar{x}) y_{1}^{m_{1}} \cdots y_{r}^{m_{r}} .
$$

Clearly, if, for a certain substitution $\bar{x} \leftarrow \bar{u}\left(\in K^{n}\right)$, all the $f_{\bar{m}}$ 's vanish, then $U^{\prime}+K \bar{u} \subseteq V(f)$. If, in addition, $\bar{u} \notin U^{\prime}$, then $U=U^{\prime}+K \bar{u}$ will be as required.

So consider the system of equations in $x_{1}, \ldots, x_{n}$ over $K$;

$$
f_{\bar{m}}(\bar{x})=0 \text { for all } \bar{m}=\left(m_{1}, \ldots, m_{r}\right), m_{i} \geq 0, \sum m_{i} \leq d .
$$

We have to show that it has a solution in $K^{n} \backslash U^{\prime}$.

First, observe that by substituting $\bar{x}=\overline{0}$ in (1), we can conclude that the polynomial $g\left(y_{1}, \ldots, y_{r}\right)=\sum f_{\bar{m}}(\overline{0}) y_{1}^{m_{1}} \cdots y_{r}^{m_{r}}$ defines the zero function $K^{r} \rightarrow$ $K$ (since $\left.U^{\prime} \subseteq V(f)\right)$. Its degree is less than or equal to $d$, and is therefore strictly less than $|K|$. It follows that $g=0$, so $f_{\bar{m}}(\overline{0})=0$ for all $\bar{m}$. This shows that system (2) has at least one solution, namely-the zero one. Now, observe that $\operatorname{deg}\left(f_{\bar{m}}\right) \leq m_{r}$. Therefore, if $D$ denotes the sum of the degrees of equations (2), then $D \leq \sum_{\bar{m}} m_{r}=\sum_{M} \operatorname{deg}_{y_{r}}(M)$, where $M$ ranges over the set of all the $\left(\begin{array}{c}r+d \\ d\end{array}\right)$ monomials of degree $\leq d$ in $y_{1}, \ldots, y_{r}$, denoted here by $\operatorname{Mon}(d, r)$. It follows that

$$
D \leq \sum_{M \in \operatorname{Mon}(d, r)} \operatorname{deg}_{y_{r}}(M)=\left(\begin{array}{l}
r+d \\
d-1
\end{array}\right),
$$

where the equality on the right can be easily verified. By Warning's second 
theorem, system (2) has at least $q^{n-D}$ solutions, where $|K|=q$. The last calculation and the choice of $n$ yield $n-D \geq n-\left(\begin{array}{c}r+d \\ d-1\end{array}\right) \geq r$, so that $q^{n-D} \geq$ $q^{r}>\left|U^{\prime}\right|$. Therefore one of these solutions must lie outside $U^{\prime}$. This completes the proof of the right inequality.

For the left one we use a simple probabilistic argument.

Note that a polynomial $f=f\left(x_{1}, \ldots, x_{n}\right)$ over $K$ of degree $\leq d<q$ vanishes on the linear space $U$ defined by $x_{r+1}=x_{r+2}=\cdots=x_{n}=0$ if and only if all its $\left(\begin{array}{c}r+d \\ d\end{array}\right)$ coefficients of the monomials in $x_{1}, \ldots, x_{r}$ of degree $d$ or less vanish. This event occurs with probability $q^{-\left(\begin{array}{c}r+d \\ d\end{array}\right)}$ in the space of all the $q^{\left(\begin{array}{c}n+d \\ d\end{array}\right)}$ possible polynomials $f$. Obviously, this probability does not change if one replaces $U$ with any other $r$-dimensional affine space. Now, if $n=N_{K}(d, r)$, then by definition every such polynomial $f$ will vanish on a certain $r$-dimensional affine space, provided it has a zero. Since the probability of $f$ having a zero is clearly greater than or equal to $q^{-1}$, we obtain

$$
A_{K}(n, r) \cdot q^{-\left(\begin{array}{c}
r+d \\
d
\end{array}\right)} \geq q^{-1}
$$

where $A_{K}(n, r)$ denotes the number of $r$-dimensional affine subspaces of $K^{n}$. It is well known that

$$
A_{K}(n, r)=q^{n-r} \cdot \frac{\left(q^{n}-1\right) \cdots \cdots\left(q^{n}-q^{r-1}\right)}{\left(q^{r}-1\right) \cdots \cdots\left(q^{r}-q^{r-1}\right)}<q^{n(r+1)-r^{2}} .
$$

We conclude that $q^{n(r+1)-r^{2}} \cdot q^{-\left(\begin{array}{c}r+d \\ d\end{array}\right)}>q^{-1}$, so that $n(r+1)>\left(\begin{array}{c}r+d \\ d\end{array}\right)+r^{2}-1$, and $n>\frac{1}{r+1}\left(\begin{array}{c}r+d \\ d\end{array}\right)+\frac{r^{2}-1}{r+1}=\frac{1}{d}\left(\begin{array}{c}r+d \\ d-1\end{array}\right)+r-1$, as required.

Remark. The upper bound given in Theorem 2 remains valid without the restriction on $d$. To see this, one should reduce the right-hand side of expression (1) (using the identities $y_{i}^{q}=y_{i}$ ), and form the corresponding system of equations. Clearly, this system must have a solution (the zero one), and its sum of degrees is less than or equal to $D$-the sum of degrees of (2)-which is in turn bounded above by $\left(\begin{array}{c}r+d \\ d-1\end{array}\right)$. This yields the desired conclusion.

Corollary. For fixed $d$ we have $M_{K}(d, r) \leq O\left(r^{(d-1) !}\right)$.

Proof. Combine the last remark with the proof of Theorem 1, showing that $M_{K}(1, r)=r+1$ and $M_{K}(d, r) \leq N_{K}\left(d, M_{K}(d-1, r)\right) \quad(d \geq 2)$.

This bound is clearly much larger than the one appearing in Ax's divisibility theorem, which is linear in $r$. However, if $d \geq 2$, one cannot expect $M_{K}(d, r)$ (and even $N_{K}(d, r)$ ) to be linear in $r$, as shown in Theorem 2. Nevertheless, it would be interesting to understand the asymptotic behaviour of $M_{K}(d, r)$.

\section{ACKNOWLEDGMENT}

I am grateful to D. Kazhdan for stimulating discussions. 


\section{REFERENCES}

1. A. Adolphson and S. Sperber, p-adic estimates for exponential sums and the theorem of Chevalley-Warning, Ann. Sci. École Norm. Sup. (4) 20 (1987), 545-556.

2. J. Ax, Zeros of polynomials over finite fields, Amer. J. Math. 86 (1964), 255-261.

3. R. Brauer, A note on systems of homogeneous algebraic equations, Bull. Amer. Math. Soc. 51 (1945), 749-755.

4. C. Chevalley, Démonstration d'une hypothèse de M. Artin, Abh. Math. Sem. Univ. Hamburg 11 (1936), 73-75.

5. M. J. Greenberg, Lectures on forms in many variables, Benjamin, Amsterdam and New York, 1969.

6. N. M. Katz, On a theorem of Ax, Amer. J. Math. 93 (1971), 485-499.

7. W. M. Schmidt, Equations over finite fields, Lecture Notes in Math., vol. 536, SpringerVerlag, Berlin and New York, 1976.

8. D. Wan, An elementary proof of a theorem of Katz, Amer. J. Math. 111 (1989), 1-8.

9. E. Warning, Bemerkung zur vorstehenden Arbeit von Herrn Chevalley, Abh. Math. Sem. Univ. Hamburg 11 (1936), 76-83.

Institute of Mathematics and Computer Sciences, The Hebrew University, Jerusalem 91904, ISRAEL

Current address: Mathematical Institute, University of Oxford, 24-29 St. Giles, Oxford 0X1 3LB, United Kingdom 